Pacific Journal of Mathematics

A REPRESENTATION THEORY FOR A CLASS OF PARTIALLY

A. HAYes 


\section{A REPRESENTATION THEORY FOR A CLASS OF PARTIALLY ORDERED RINGS}

\section{Allan Hayes}

The lattice ordered rings known as $f$-rings, introduced by Birkhoff and Pierce in [1], have been studied very intensively in the last few years. In particular Pierce has shown in [4] that the $f$-rings without nonzero nilpotents are precisely the (isomorphic images of) lattice ordered subdirect unions of totally ordered rings with integrity, and Johnson in [2] has gone on to prove that any Archimedean $f$-ring with no nonzero nilpotents can be represented as a lattice ordered ring of continuous extended realvalued functions on a locally compact Hausdorff space.

Since many commonly occurring examples of partially ordered rings are not lattice ordered it is natural to ask whether these two results can be generalised so as to be independent of the lattice structure. Such a generalisation is given here when multiplication is assumed commutative.

Theorem 1 characterises the subdirect unions of totally ordered commutative rings with integrity; Theorem 2 sharpens this result and Theorem 3 completes the programme by extending Johnson's representation.

The plan of the paper is as follows:

Section 1 is an introduction to the subject matter and methods of the paper; the succeeding three sections contain proofs of Theorems 1,2 and 3 respectively and 85 shows that for $f$-rings the representations given preserve the lattice structure.

1. Introduction. Throughout this paper "ring" will be an abbreviation for "commutative associative ring".

A partially ordered (or po-) ring is a ring whose elements are partially ordered in such a way that if $a \geqq b$ then $a+c \geqq b+c$ for all $c$ and $a c \geqq b c$ for all $c \geqq 0$. Among the po-rings those with integrity (i.e. without divisors of zero) and a total ordering (the toi-rings) are particularly simple and it is our first aim to find out when a poring can suitably be built up from toi-rings. To make this more precise:

If $\left\{R_{i}\right\}_{i \in I}$ is a nonempty family of toi-rings their direct union, $\sum R_{i}$, is formed by taking the class of all functions $a: I \rightarrow \bigcup R_{i}$ with $a(i) \in R_{i}$ for all $i$, and defining addition by $(a+b)(i)=a(i)+b(i)$ for all $i$; multiplication by $(a b)(i)=a(i) b(i)$ for all $i$, and order by $a \geqq b$

Received September 16, 1963. This work was supported by N.S.F. Grant 3639-508476. 
when $a(i) \geqq b(i)$ for all $i . \quad \sum R_{i}$ is then a po-ring (in fact it is an $f$-ring). A subdirect union of the family $\left\{R_{i}\right\}_{i \in I}$ is a subring, $R$, of $\sum R_{i}$ satisfying $R(i)=R_{i}$ for all $i$, together with the partial ordering induced on it by the partial ordering of $\sum R_{i}$. If in addition, whenever $R$ contains $a$ it contains $a^{+}$, defined by $a^{+}(i)=a(i) \vee 0$ for all $i$, it is called a lattice ordered subdirect union of $\left\{R_{i}\right\}_{i \in I}$ (and is an $f$-ring).

A mapping, $h$, from one po-ring to another is called a homomorphism if it is a ring homomorphism such that $h(a) \geqq h(b)$ when $a \geqq b$ : it is called an isomorphism if it is a ring isomorphism with $h(a) \geqq h(b)$ if and only if $a \geqq b$.

Suppose $R$ is a po-ring and $\mathfrak{S}$ is a nonempty class of homomorphisms, $h$, of $R$ onto toi-rings $R_{h}$ respectively. Suppose further that if $a \in R$ and $a \geqq 0$ then there is an $h \in \mathfrak{S}$ with $h(a)<0$. For any $a \in R$ let $\widetilde{a}$ be the function on $\mathfrak{S}$ defined by $\widetilde{a}(h)=h(\alpha)$ for all $h \in \mathfrak{S}$. Then $\widetilde{R}=$ $\{\widetilde{a}: a \in R\}$, with the natural induced structure, is a subdirect union of $\sum R_{h}$, and the map $a \rightarrow \widetilde{a}$ is an isomorphism of $R$ onto $\widetilde{R}$.

To generate the homomorphisms needed we look at the semirings in $R$ (i.e. the nonempty subsets, $S$, of $R$ with $S S \cup(S+S) \subset S$ ). Under conditions stated in the next section, if $a_{0} \geqq 0$ then maximalisation by Zorn's Lemma yields a semiring $P$, with $a_{0} \notin P$ and $P^{\prime} P^{\prime} \subset-P^{\prime},{ }^{1}$ which contains all $a \geqq 0$ and all squares in $R$. From this a homomorphism onto a toi-ring arises as follows:

$I=P \cap-P$ is a prime ring ideal in $R$. For,

(i) if $a, b \in I$ then clearly $a-b \in I$;

(ii) if $a \in I$ and $c \in R$ then $c \in P$ or $c \in-P$ (otherwise $-((-c) c)=$ $\left.c^{2} \in P^{\prime}\right)$ and in either case $a c \in I$;

(iii) if $a \in I^{\prime}$ and $b \in I^{\prime}$ then $a \in P^{\prime}$ or $-a \in P^{\prime}$ and $b \in P^{\prime}$ or $-b \in P^{\prime}$; whence $a b \in P^{\prime}$ or $-a b \in P^{\prime}$ and certainly $a b \in I^{\prime}$. Let $h$ be the canonical homomorphism of $R$ onto $R / I$, which is a ring with integrity. A simple calculation shows that $h(P)$ is a semiring, $h^{-1}(h(P))=P, h(P) \cup-h(P)=$ $h(R)$ and $h(P) \cap-h(P)=\{0\}$. So if we define $h(a) \geqq h(b)$ to mean $h(a)-h(b) \in h(P)$, (i.e. $a-b \in P$ ) then this is a total ordering making $R / I$ into a toi-ring which is called the quotient ring of $R$ by $P$ and is denoted by $R / P$. Since $P$ contains all $a \geqq 0, a_{0} \in P^{\prime}$ and $h^{-1}(h P)=$ $P, h$ is a homomorphism of $R$ onto $R / P$ and $h\left(a_{0}\right)<0$.

It is convenient to write $a(P)$ for $h(a)$ and to use abbreviations similar to writing $a \geqq b,(P)$ for $a(P) \geqq b(P)$.

The representation of a po-ring as a ring of real valued functions on some set would be very useful. Unfortunately it seems difficult to find a simple general condition permitting this, which does not make all the functions used bounded. Nevertheless, a po-ring of the type here considered which is also Archimedean (that is $n a \leqq b, n=$ 
$1,2, \cdots$ implies $a \leqq 0$ ) can be represented using functions with values in the extended real numbers. The possibility of this is suggested by the observation that in a toi-ring $R$ if $a b \geqq 0$ and $a>0$ then $c b \geqq 0$ for all $c \geqq 0$ so that if

$$
\begin{aligned}
& \bar{a}=\inf \{m / n: m \text { and } n \text { are integers, } n>0, \text { and } \\
& m b \geqq n a b \text { for all } b>0\} \\
& (=\sup \{m / n: m \text { and } n \text { are integers, } n>0, \text { and } \\
& m b \leqq n a b \text { for all } b>0\})
\end{aligned}
$$

it follows by routine calculations that $\bar{a} \geqq 0$ when $a \geqq 0, \overline{a b}=\overline{a b}$ unless $\bar{a}=0$ and $\bar{b}= \pm \infty$ or vice versa, and $\overline{a+b}=\bar{a}+\bar{b}$ unless $\bar{a}$ and $\bar{b}$ are infinite and of opposite sign. Here the infimum is taken in the extended reals and the infimum of the empty set is $+\infty$. The main problem is to guarantee that the substitution of $\bar{a}$ for $a$, which is usually far from being $(1-1)$, still leaves enough information for reconstruction of the original po-ring; it is here that the assumption that the ring is Archimedean is required.

The following notation will be standard for the rest of the paper:

If $R$ is a po-ring then $R^{+}=\{x: x \geqq 0\}$ is the class of quasi positive elements of $R$ and $R^{++}=\{x: x>0\}$ is the class of positive elements of $R$.

$Z$ is the po-ring of integers.

$\boldsymbol{R}$ is the po-ring of real numbers and $\overline{\boldsymbol{R}}$ the "quasi po-ring" of the extended real numbers with the usual topology of the two point compactification.

If a set $X$ is fixed in some context and $Y \subset X$ then $Y^{\prime}$ will denote $X \backslash Y$. The empty set is denoted by $\phi$. The set with $x$ as its only element will sometimes be denoted simply by $x$.

If $A$ and $B$ are subsets of a partially ordered set then $A \leqq B$ means that every element of $A$ is less than or equal to every element in $B$.

2. $f^{*}$-rings. Lemma 1 below, on the semirings in a ring, is the key to the rest of the paper. It is used in this section to produce a characterisation of the isomorphic images of subdirect sums of to $i$-rings (Theorem 1).

A semiring $S$ in a ring $R$ is said to be normal with respect to a nonempty subset $H$ of $R$ if no expression of the form

$$
\sum_{i=1}^{N}(-1)^{n_{i}+1} s_{i} a_{i, 1} a_{i, 2} \cdots a_{i, n_{i}}-a_{1} a_{2} \cdots a_{2 q}-s
$$


is zero, where each $a$ is in $H$, each $s$ is in $S$, each $n$ is in $Z^{++}, q$ is in $\boldsymbol{Z}^{++}$and $N$ is in $\boldsymbol{Z}^{+}$.

If $S$ contains all squares in $R$ and $H=\{a\}$ then $S$ is normal with respect to $H$ if and only if $s a-a^{2 n} \in S^{\prime}$ for all $s \in S$ and all $n \in Z^{++}$.

Normality of $S$ with respect to $H$ implies $H \subset S^{\prime}$. For if $a \in H \cap S$ then $(-1)^{1+1} a \alpha+(-1)^{1+1} a \alpha-a \alpha-a \alpha=0$.

A semiring $P$ in a ring $R$ is called prime if $P^{\prime} P^{\prime} \subset-P^{\prime}$.

The usefulness of normality is due to the following result:

LEMmA. If $S$ is a semiring containing all squares in a ring $R$, and $H$ is a nonempty subset of $R$ then there is a prime semiring $P$ in $R$ with $P \supset S$ and $P^{\prime} \supset H$ if and only if $S$ is normal with respect to $H$.

Proof. (i) If such a $P$ exists then for any $a_{1}, a_{2}, \cdots, a_{n} \in P^{\prime}$ and any $s \in S,(-1)^{n+1} s a_{1} a_{2} \cdots a_{n} \leqq 0,(P)$ (see $\S 1$ for this notation); and if $n$ is even $-a_{1} a_{2} \cdots a_{n}<0,(P)$. So any expression of the form $(1)$ is $<0,(P)$ and cannot be equal to zero.

(ii) Conversely, if $S$ is normal with respect to $H$ then Zorn's lemma shows that there is a maximal semiring, $P$, among the semirings containing $S$ which are normal with respect to $H$. It will be proved that $P$ is as required.

Since $P$ contains all squares in $R$, if $x \in R$ then the semiring, $P_{x}$, generated by $P \cup\{x\}$ is $Z^{+} x+x P+P$. So if $x \in P^{\prime}$ and $y \in P^{\prime}$, since neither $P_{x}$ nor $P_{y}$ is normal with respect to $H$, there are identities of the form

$$
\sum_{i=1}^{N}(-1)^{n_{i}+1}\left(s_{i}^{\prime}+s_{i}\right) a_{i, 1} \cdots a_{i, n_{i}}-a_{1} a_{2} \cdots a_{2 q}-\left(s^{\prime}+s\right)=0
$$

and

$$
\sum_{j=1}^{M}(-1)^{m_{j}+1}\left(t_{j}^{\prime}+t_{j}\right) b_{j, 1} \cdots b_{j, m_{j}}-b_{1} b_{2} \cdots b_{2 r}-\left(t^{\prime}+t\right)=0,
$$

where every $a$ and $b$ is in $H$, every $n$ and $m$ is in $Z^{++}, q$ and $r$ are in $Z^{++}, M$ and $N$ are in $Z^{+}$, every $s$ and $t$ is in $P$, every $s^{\prime}$ is in $\boldsymbol{Z}^{+} x+x P$ and every $t^{\prime}$ is in $\boldsymbol{Z}^{+} y+y P$.

Collection of the terms involving $x, y$ respectively to one side of the equations (taking the rest to the other side) followed by multiplication of the new equalities yields, after rearrangement, the following, 


$$
\begin{aligned}
\sum_{i=1 j=1}^{N M} & (-1)^{n_{i}+m_{j}+2} s_{i}^{\prime} t_{j}^{\prime} a_{i, 1} \cdots a_{i, n_{i}} b_{j, 1} \cdots b_{j, m_{j}}+s^{\prime} t^{\prime} \\
& +\sum_{i=1}^{N}(-1)^{n_{i}+2} s_{i}^{\prime} t^{\prime} a_{i, 1} \cdots a_{i, n_{i}}+\sum_{j=1}^{M}(-1)^{m_{j}+2} t_{j}^{\prime} s^{\prime} b_{j, 1} \cdots b_{j, m_{j}} \\
& +\sum_{i=1 j=1}^{N M}(-1)^{n_{i}+m_{j}+1} s_{i} t_{j} a_{i, 1} \cdots a_{i, n_{i}} b_{j, 1} \cdots b_{j, m_{j}} \\
& +\sum_{i=1}^{N}(-1)^{n_{i}+1} s_{i} t a_{i, 1} a_{i, 2} \cdots a_{i, n_{i}} \\
& +\sum_{i=1}^{N}(-1)^{n_{i}+2 r+1} s_{i} a_{i, 1} \cdots a_{i, n} b_{1} \cdots b_{2 r} \\
& +\sum_{j=1}^{M}(-1)^{m_{j}+1} t_{j} s b_{j, 1} \cdots b_{j, m_{j}} \\
& +\sum_{j=1}^{M}(-1)^{m_{j}+2 q+1} t_{j} b_{j, 1} \cdots b_{j, m_{j}} a_{1} \cdots a_{2 q}+(-1)^{2 r+1} s b_{1} \cdots b_{2 r} \\
& +(-1)^{2 q+1} t a_{1} \cdots a_{2 q}-s t-a_{1} a_{2} \cdots a_{2 q} b_{1} b_{2} \cdots b_{2 r}=0 .
\end{aligned}
$$

If $x y \in-P$ this would contradict the hypothesis that $P$ is normal with respect to $H$.

It is clear that $P \supset S$ and $P^{\prime} \supset H$, so the proof is complete.

Corollary. If $H$ has only one element, a, then there is a $P$ as required if and only if $s a-a^{2 n} \in S^{\prime}$ for all $s \in S$ and all $n \in \boldsymbol{Z}^{++}$.

The full force of Lemma 1 is not required until $\S 4$; up to that point the corollary will be sufficient.

From now on $A$ will always denote a po-ring, $\mathscr{S}$ the class of all semirings in $A$ which contain $A^{+}$and $\mathscr{P}$ the class of prime semirings in $A$ which contain $A^{+}$. If $\mathscr{D}$ is a subset of $\mathscr{P}$ such that for any $a \notin A^{+}$there is a $D \in \mathscr{D}$ with $a(D)<0$ then $\mathscr{D}$ will be said to be distinguishing.

$A$ is called an $f^{*}$-ring if $A^{+}$contains all squares in $A$ and is normal with respect to every single point set $\{a\}$ with $a \notin A^{+}$.

We have:

THEOREM 1. A is isomorphic to a subdirect union of toi-rings if and only if it is an $f^{*}$-ring.

Proof. (i) If $A$ is an $f^{*}$-ring then the Corollary to Lemma 1 shows that $\mathscr{P}$ is distinguishing, so that from the discussion in the previous section, $A$ is isomorphic to a subdirect union of $t o i$-rings $\{A / P\}_{P \in \mathscr{P}}$.

(ii) If $A$ can be identified with a subdirect union $R$ of toi-rings $\left\{R_{i}\right\}_{i \in I}$ then $a \in A \backslash A^{+}$implies $a(i)<0$ for some $i \in I$, say $a\left(i_{0}\right)<0$. Consequently, if $s \in A^{+}$and $n \in Z^{++},\left(s a-a^{2 n}\right)\left(i_{0}\right)<0$ and $s a-a^{2 n} \notin A^{+}$. 
Thus $A$ is normal with respect to $\{a\}$. Also, for any $a \in A,\left(a^{2}\right)(i)=$ $a(i)^{2} \geqq 0$ for all $i \in I$, so $a^{2} \in A^{+}$. Thus $A$ is an $f^{*}$-ring.

3. Ring Archimedean $f^{*}$-rings. In this section a class of $f^{*}$ rings is introduced which includes the Archimedean $f^{*}$-rings and for which a considerable sharpening of Theorem 1 is possible (see Theorem 2 below).

A po-ring $R$ is called ring (or $r$-) Archimedean if $Z^{+} a+R^{+} a \leqq b$ implies $a \leqq 0$. An Archimedean po-ring is necessarily $r$-Archimedean, but the converse is not true, since every totally ordered field is $r$ Archimedean.

The following two measures of size will be used.

In any to $i$-ring $R$ an element, $a$, is called a ring $(r-)$ order unit if $Z^{+} a+R^{+} a-R^{+}=R$, and is called ring $(r-)$ infinitesimal if $\boldsymbol{Z}^{+} a^{2}+R^{+} a^{2} \leqq|a|$. Notice that if for some $q>0,\left(\boldsymbol{Z}^{+}|a|+R^{+}|a|\right) q \leqq q$ then $a$ is $r$-infinitesimal and $\left(Z^{+}|a|+R^{+}|a|\right) p \leqq p$ for all $p \geqq 0$. A to $i$-ring is $r$-Archimedean if and only if every positive element is an $r$-order unit.

The main result to be proved is:

THEOREm 2. A necessary and sufficient condition that $A$ be an $r$-Archimedean $f^{*}$-ring is that it be isomorphic to a subdirect union of $r$-Archimedean toi-rings with no nonzero $r$-infinitesimal elements.

It will be convenient to divide up the proof into a number of lemmas.

Lemma 2. Let $A$ be an $r$-Archimedean $f^{*}$-ring and $\mathscr{D}$ a distinguishing subclass of $\mathscr{P}$. If $a(D)$ is r-infinitesimal in $A / D$ for all $D \in \mathscr{D}$ such that $a \notin D$ then $a \geqq 0$.

Proof. For each $D \in \mathscr{D}$ either (i) $a \geqq 0$ or (ii) $a<0,(D)$ and $\left[\boldsymbol{Z}^{+}(-a)+A^{+}(-a)\right](-a) \leqq(-a),(D)$. In either case $\left[\boldsymbol{Z}^{+}(-a)+\right.$ $\left.A^{+}(-a)\right] a^{2} \leqq a^{2}(D)$. Therefore, since $\mathscr{D}$ is distinguishing, $\left[Z^{+}(-a)+\right.$ $\left.A^{+}(-a)\right] a^{2} \leqq a^{2}$; whence, $A$ being $r$-Archimedean, $(-a)^{3} \leqq 0$, and in an $f^{*}$-ring this implies $-a \leqq 0$, i.e. $a \geqq 0$.

LEMMA 3. In any toi-ring $R$ if $a$ is not $r$-infinitesimal then $|a|$ is an r-order unit.

Proof. If $Z^{+}|a|+R^{+}|a| \leqq b$ while $\left(n_{0}|a|+p_{0}|a|\right)|a|>|a|$ with $n_{0} \in Z^{+}$and $p_{0} \in R^{+}$, then $b>0$ and $\left(n_{0}|a|+p_{0}|a|\right) b>b \geqq$ $\left(n_{0} b+p_{0} b\right)|a|=\left(n_{0}|a|+p_{0}|a|\right) b$, which is impossible. 
Let $\mathscr{C}$ be the class of maximal elements in $\mathscr{P}$ (under set inclusion).

Lemma 4. If $P \in \mathscr{P}, a \in P^{\prime}$ and $|a(P)|$ is an r-order unit in $A / P$ then no $Q \in \mathscr{P}$ can contain $P \cup\{a\}$, therefore there is an $M \in \mathscr{C l}$ with $a \notin M \supset P$.

Proof. Suppose such a $Q$ does exist and take $q \in Q^{\prime}$. Since $-a(P)$ is an $r$-order unit in $A / P$ there are $n \in \boldsymbol{Z}^{+}$and $p \in P$ such that $n[(-a)+p(-a)] \geqq q(P)$. So $n(-a)+p(-a)-q \in P$ and $q \in P+n a+$ $p a \subset Q$, contrary to the hypothesis that $q \in Q^{\prime}$.

The three previous lemmas show that $\mathscr{C}$ is distinguishing for $r$-Archimedean $f^{*}$-rings. However, a stronger result is needed to prove the Theorem.

LEMMA 5. In any toi-ring $R$ the class, $I$, of r-infinitesimal elements is a prime ring ideal such that if $|c| \leqq|a|$ and $a \in I$ then $c \in I$.

Proof. If $a \in I$ and $|c|<|a|$, then for any $n \in Z^{+}$and $p, q \in R^{+}$, $(n|c|+p|c|) q \leqq(n|a|+p|a|) q \leqq q$, so $c \in I$.

If $a, b \in I, \quad n \in Z^{+}$and $p, q \in R^{+},(2 n|a-b|+2 p|a-b|) q \leqq$ $(2 n|a|+2 p|a|) q+(2 n|b|+2 p|b|) q \leqq 2 q$, whence $(n|a-b|+p|a-b|) q \leqq q$ and $a-b \in I$.

If $a \in I$ and $e \in R$ then $a e \in I$, for if not then, by Lemma 3 , there are $n \in Z^{+}$and $p \in R^{+}$such that $n|a e|+p|a e|>2|e|$. But, since $a \in I,|e| \geqq n|a e|+p|a e|$, and these two inequalities together yield the contradiction, $0>|e|$.

$I$ has now been proved to be an ideal: it remains to prove that it is prime.

If $a, b \in I^{\prime}$ there are $m, n \in Z^{+}$and $p, q \in R^{+}$such that for any $s>0,(m|a|+p|a|) s>s$ and $(n|b|+q|b|) s>s$, whence, by multiplication $(m n|a b|+(m p+n q+p q)|a b|) s^{2}>s^{2}>0$, and so $a b \in I^{\prime}$.

Let $\mathscr{C}^{*}=\{M \in \mathscr{C}: A / M$ contains no nonzero $r$-infinitesimal elements\}.

Then we have:

Lemma 6. If $M \in \mathscr{C} \backslash \mathscr{C}^{*}$ then every element of $A / M$ is $r$-infinitesimal.

Proof. Let $I_{M}=\{x \in A: x(M)$ is $r$-infinitesimal $\}$ and let $P=I_{M}+M$. Lemma 5 shows immediately that $P$ is a semiring containing $A$. Furthermore if $a, b \in P^{\prime}$ then $-a(M)$ and $-b(M)$ are positive and non$r$-infinitesimal in $A / M$. So $a(M) b(M)$ is positive and non- $r$-infinitesimal in $A / M$, and $-a b \in P^{\prime}$. 
The maximality of $M$ and the supposition that $M \notin \mathscr{C}^{*}$ imply therefore that $P=A$. So if $a \in A$ there is a $b \in I_{M}$ with $|b(M)| \geqq$ $|a(M)|$, whence $a(M)$ is $r$-infinitesimal.

The following simple result proves to be important.

LEMMA 7. If a is a non-r-infinitesimal positive element of a toi-ring $R$ then there is a $b \in R^{+}$such that $b^{2}>a$

Proof. If $a^{2} \geqq a$ there is nothing to prove. If $a^{2}<a$ then, since $a$ is not $r$-infinitesimal, there are $n \in Z^{+}$and $p \in R^{+}$with $(n a+p a) a>a$; whence $(n a+p a)^{2} a^{2}>a^{2}>a^{3},(n a+p a)^{2} a^{2}>a^{3}$ and $(n a+p a)^{2}>a$. So $n a+p a$ may be taken for $b$.

\section{Proof of Theorem 2.}

(i) Necessity. $\mathscr{C}^{*}$ is a distinguishing subset of $\mathscr{P}$; for if $a \nsupseteq 0$ Lemma 2 shows that there is a $P \in \mathscr{P}$ with $a \in P^{\prime}$ and $a(P)$ not $r$-infinitesimal and by Lemma 4 there is an $M \in \mathscr{C}$ containing $A^{+}$ with $a \notin M$, so $\mathscr{C}$ is distinguishing. Lemma 6 and a second application of Lemma 4 show that $\mathscr{M}^{*}$ is distinguishing.

Reference to the introduction completes the proof.

(ii) Sufficiency. Suppose $A$ is identified with a subdirect union of a family $\left\{R_{i}\right\}_{i \in I}$ of toi-rings without nonzero $r$-infinitesimal elements. If $a \in A$ satisfies $Z^{+} a+a A^{+} \leqq b$ and $a(i)>0$ for some $i \in I$ then $\boldsymbol{Z}^{+} a(i)+p^{2}(i) a(i) \leqq b(i)$ for all $p \in A^{+}$; and by Lemma $7, \boldsymbol{Z}^{+} a(i)+$ $R_{i}^{+} a(i) \leqq b(i)$. So, since $R_{i}$ is $r$-Archimedean, $a(i) \leqq 0$, contrary to hypothesis. Thus $a \leqq 0$ and $A$ is $r$-Archimedean.

4. Archimedean $f^{*}$-rings. A ring of $\bar{R}$-valued functions on a nonempty set $X$ is a nonempty class, $R$, of $\bar{R}$-valued functions on $X$ such that

(i) If $\left\{f_{i}\right\}_{i \in I}$ is any finite subclass of $R$ there is at least one point $x$ in $X$ where every $f_{i}(x)$ is finite.

(ii) If $f, g$ and $h$ are in $R$ and $f(x) \geqq g(x)$ for all $x$ where $h(x)$ is finite then $f(x) \geqq g(x)$ for all $x$ in $X$.

(iii) If $f$ and $g$ are in $R$ then there are functions $s, p$ and $n$ in $R$ such that $s(x)=f(x)+g(x)$ whenever $f(x)$ and $g(x)$ are not infinite and of opposite sign, $p(x)=f(x) g(x)$ unless $f(x)=0$ and $g(x)= \pm \infty$ or vice versa, and $n(x)=-f(x)$ for all $x$ in $X$.

Condition (ii) shows that such $s, p$ and $n$ are unique, so they may be denoted by $f+g, f g$ and $-f$ respectively.

Subsets of $X$ of the form $\{x: f(x)= \pm \infty\}$ are called nul-sets (a name suggested by integration theory and Condition (ii)).

It is easily seen that any ring of $\bar{R}$-valued functions on a set $X$ 
is an Archimedean $f^{*}$-ring. Conversely, if $A$ is an Archimedean $f^{*}$-ring, and for each $a \in A \bar{a}$ denotes the function $P \rightarrow \overline{a(P)}$ defined on $\mathscr{P}(\overline{a(P})$ was defined in the Introduction), then Lemma 8 below and the remarks in the Introduction show that for any distinguishing subset $\mathscr{D}$ of $\mathscr{P} \bar{A} \mid \mathscr{D}=\{\bar{a} \mid \mathscr{D}: a \in A\}$ is a ring of $\bar{R}$-valued functions on $\mathscr{D}$, and the map $a \rightarrow \bar{a} \mid \mathscr{D}$ is an isomorphism of $A$ onto $\bar{A} \mid \mathscr{D}$.

If $\mathscr{D}$ is any subset of $\mathscr{P}, a, b \in A$ and $\lambda \in \bar{R}$ it is convenient to adopt conventions similar to $\mathscr{D}(\bar{a} \geqq \lambda)$ for $\{D \in \mathscr{D}: \overline{a(D)} \geqq \lambda\}$ and $\mathscr{D}(a \geqq b)$ for $\{D \in \mathscr{D}: a(D) \geqq b(D)\}$.

Lemma 8. If $A$ is an Archimedean $f^{*}$-ring and $\mathscr{D}$ is a distinguishing subset of $\mathscr{P}$ and if $\mathscr{D}(\bar{a}<\bar{b})$ is a nul-set then $a \geqq b$.

Proof. There is a $c \in A^{+}$with $\mathscr{D}(\bar{c}=\infty) \supset \mathscr{D}(\bar{a}<\bar{b})$; so $e \stackrel{\text { def }}{=}$ $c+a^{2}+b^{2}$ satisfies $\mathscr{D}(a \neq 0) \cup \mathscr{D}(b \neq 0) \subset \mathscr{D}(e \neq 0)$ and $\mathscr{D}(\bar{a}<\bar{b}) \cup$ $\mathscr{D}(\bar{a}= \pm \infty) \cup \mathscr{D}(\bar{b}= \pm \infty) \subset \mathscr{D}(\bar{e}=\infty)$.

Consider the following three situations which may occur for a $D \in \mathscr{D}:$

(i) $b>a,(D)$ and $\bar{e}(D)=\infty$; whence $Z^{+}(b-a) \leqq e(b-a),(D)$ and so $\boldsymbol{Z}^{+}(b-a) 2 e \leqq e^{4}+(b-a)^{2},(D)$.

(ii) $b>a,(D)$, and $\bar{e}(D)<\infty$; whence $\bar{a}(D)$ and $\bar{b}(D)$ are finite, $(\overline{b-a})(D)=0$, and so $Z^{+}(b-a) 2 e \leqq 2 e,(D)$.

(iii) $b \leqq a,(D)$.

In all cases $\boldsymbol{Z}^{+}(b-a) \leqq e^{4}+(b-a)^{2}+2 e,(D)$. So $\boldsymbol{Z}^{+}(b-a) e \leqq$ $e^{4}+(b-a)^{2}+2 e$ and, $A$ being Archimedean, $(b-a) e \leqq 0$. This, in an $f^{*}$-ring with $e$ as here defined, implies $b-a \leqq 0$, that is $a \geqq b$.

Corollary. No nul-set can contain a nonempty set of the form $\mathscr{D}(\bar{a}>0)$.

Let $\mathscr{C}^{* *}=\left\{M \in \mathscr{C}^{*}: \exists a \in A\right.$ with $\bar{a}(M)$ nonzero $\}$.

Lemma 8 shows that $\mathscr{C}^{* *}$ is distinguishing and so the mapping $a \rightarrow \bar{a} \mid \mathscr{C}^{* *}$ is an isomorphism of $A$ onto $\bar{A} \mid \mathscr{C}^{* *}$.

Two natural topologies for $\mathscr{C}^{* *}, \mathscr{T}_{1}$ with the sets of the form $\mathscr{C}^{* *}(a>0)$ as a subbase, and $\mathscr{T}_{2}$ with the sets of the form $\mathscr{C}^{* *}(\bar{a}>0)$ as a subbase, turn out to be the same.

Lemma 9. $\mathscr{T}_{1}=\mathscr{T}_{2}(=\mathscr{T}$ say). $\mathscr{T}$ is Hausdorff and is the weak topology induced on $\mathscr{C l}^{* *}$ by $\bar{A}$.

Proof. $\mathscr{T}_{2} \supset \mathscr{T}_{1}$, for if $M \in \mathscr{C}^{* *}(a>0)$ there is a $b \in A$ with $\bar{b}(M)>0$, and since $a(M)$ is an $r$-order unit, there are, using Lemma 7, $n \in \boldsymbol{Z}^{+}$and $e \in A^{+} \quad$ such that $n a+e^{2} a>b,(M)$. So $M \in \mathscr{C}^{* *}\left(\overline{n a+e^{2} a}>0\right) \subset \mathscr{C}^{* *}\left(n a+e^{2} a>0\right) \subset \mathscr{L}^{* *}(a>0)$. Conversely, $\mathscr{T}_{1} \supset \mathscr{T}_{2}$, for if $M \in \mathscr{C}^{* *}(\bar{a}>0)$ then for some $n \in \boldsymbol{Z}^{++}$, 
$M \in \mathscr{L}^{* *}(\bar{a}>1 / n) ;$ so $n a^{3}>a^{2},(M)$ and $M \in \mathscr{C}^{* *}\left(n a^{3}-a^{2}>0\right) \subset$ $\mathscr{C}^{* *}(\bar{a} \geqq 1 / n) \subset \mathscr{C}^{* *}(\bar{a}>0)$. $\mathscr{T}$ is Hausdorff. If $M_{1}, M_{2} \in \mathscr{C}^{* *}$ and $M_{1} \neq M_{2}$ there are $a_{1} \in M_{1} \backslash M_{2}$ and $a_{2} \in M_{2} \backslash M_{1}$. Whence $a=a_{1}$ $a_{2} \in\left(-M_{1}^{\prime}\right) \cap M_{2}^{\prime}$, that is $M_{2} \in \mathscr{M}^{* *}(a<0)$ and $M_{1} \in \mathscr{M}^{* *}(a>0)$.

Finally, $\mathscr{T}$ is the weak topology induced by $\bar{A}$ on $\mathscr{C}^{* *}$. For, by definition, $\mathscr{T}$ is coarser than this weak topology. Conversely, if $\lambda>-\infty \mathscr{C}^{* *}(\bar{a} \geqq \lambda)=\cap\left\{\mathscr{C}^{* *}\left(\bar{s} \bar{e}^{2} \geqq r \bar{e}^{2}\right): r / s<\lambda, s>0\right.$ and $\left.e \in A\right\}$, and so is closed with respect to $\mathscr{T}$.

Next it is shown that $\mathscr{C}^{* *}(\bar{a} \geqq \varepsilon)$ is compact for all $\varepsilon>0$ and all $a \in A$.

It is sufficient to prove the following result.

Lemma 10. If $a \in A$ then $\mathscr{C}^{* *}(\bar{a} \leqq-1)$ is compact.

Proof. Alexander's Theorem ([3] p. 139) shows that it is sufficient. to prove that any cover of $\mathscr{C}^{* * *}(\bar{a} \leqq-1)$ by sets of the form $\mathscr{C}^{* *}(c<0), c \in A$, has a finite subcover.

Accordingly, suppose $C$ is a subset of $A$ such that $\left\{\mathscr{C}^{* *}(c<0)\right.$ : $c \in C\}$ covers $\mathscr{C}^{* *}(\bar{a} \leqq-1)$ and contains no finite subcover. A contradiction will be derived from this.

Consider any $M \in \mathscr{C}^{* *}(\bar{a} \leqq-1)$ and any rational number $m / n$ with $n>0, m>2$ and $2 / 3<m / n<1$. Since $\bar{a}(M) \leqq-1, n a a^{4}<-m a^{4},(M)$. so $n a a^{4}+(m-2) a^{4}<-2 a^{4}<-a^{2},(M)$, that is $\left[n a \cdot a^{2}+(m-2) a^{2}\right] a^{2}+$ $a^{2}<0,(M)$. Thus $\left[n a \cdot a^{2}+(m-2) a^{2}\right] a^{2}+a^{2} \in N=\cap\left\{M^{\prime}: \bar{a}(M) \leqq-1\right\}$.

Let $K=\left\{n a \cdot a^{2}+(m-2) a^{2}: m \geqq 2, n>0\right.$ and $\left.2 / 3<m / n<1\right\}$.

If $\left\{c_{i}\right\}_{i=1}^{r} \subset C$ there is an $M \in \mathscr{C}^{* *}(\bar{a} \leqq-1)$ with $\left\{c_{i}\right\}_{i=1}^{r} \subset M$. So the semiring, $S$, generated by $A^{+} \cup C$ is normal with respect to $N$ and there is a $P \in \mathscr{P}$ with $P \supset S$ and $P \cap N=\phi$. For any $k \in K$, $k a^{2}+a^{2}<0,(P)$, so $k(P)$ is not $r$-infinitesimal in $A / P$. There is therefore an $M_{0} \in \mathscr{C}$ with $M_{0} \cap K=\phi$. Now for any element $n a \cdot a^{2}+$ $(m-2) a^{2}$ of $K n a \cdot a^{2}+(m-2) a^{2}<0,\left(M_{0}\right)$; whence $\bar{a}\left(M_{0}\right) \leqq-(m-2) / n$. Consequently $\bar{a}\left(M_{0}\right) \leqq-1$, so $M_{0} \in \mathscr{C}^{* *}$, while $M_{0} \supset C$, which is: contrary to the hypothesis on $C$.

$\mathscr{C}^{* *}$ may include semirings $M$ such that $\bar{A}(M) \subset\{0, \pm \infty\}$. Lemma 8 shows that these are not algebraically significant (i.e. $\mathscr{C}^{* * *} \stackrel{\text { def }}{=}$ $\left\{M \in \mathscr{C}^{* *}: \exists a \in A\right.$ with $\left.\bar{a}(M) \notin\{0, \pm \infty\}\right\}$ is distinguishing). Considered as a subspace of the topological space $\left\{\mathscr{C}^{* *}, \mathscr{T}\right\}, \mathscr{C}^{* * *}$ is a Hausdorff space. Further, since for all $a \in A$ and all $\lambda, \varepsilon \in R^{+}, \mathscr{C}^{* *}(\lambda \geqq \bar{a} \geqq \varepsilon)$ is a closed, and therefore compact, subset of $\left\{\mathscr{C}^{* *}, \mathscr{T}\right\}$ which is. contained in $\mathscr{M}^{* * *}$. So $\mathscr{M}^{* * *}$ is a locally compact Hausdorff space; for if $D \in \mathscr{C}^{* * *}$ and $D \in \mathscr{M}^{* *}(\bar{a}>0)$ there is a $b \in A$ with $\infty>\bar{b}(D)>0$, so $\mathscr{C}^{* *}(\bar{a} \geqq 1 / 2 \bar{a}(D) \wedge 1) \cap \mathscr{C}^{* *}(2 \bar{b}(D) \geqq \bar{b} \geqq 1 / 2 \bar{b}(D))$ is a compact. neighbourhood of $D$ in $\mathscr{C}^{* * *}$. 
The following analogue of [2] Theorem 4.1 has now been proved.

TheOREm 3. If $A$ is an Archimedean $f^{*}$-ring the mapping $a \rightarrow$ $\bar{a} \mid \mathscr{C}^{* * *}$ is an isomorphism of $A$ onto a ring $\bar{A} \mid \mathscr{C}^{* * *}$ of extended real valued functions on $\mathscr{C l}^{* * *}$. The weak topology induced on $\mathscr{C}^{* * *}$ by $\bar{A} \mid \mathscr{C}^{* * *}$ is Hausdorff and locally compact and relative to it each set $\mathscr{C}^{* * *}(\lambda \geqq \bar{a} \geqq \varepsilon)$ with $a \in A$ and $\lambda, \varepsilon \in \boldsymbol{R}^{++}$is compact. No function is infinite at every point of a nonempty set of the form $\mathscr{C}^{* * *}(\bar{a}>0)$.

The rest of Johnson's theorem seems to require that $A$ be an $f$-ring.

5. $f$-rings. A commutative $f$-ring is a po-ring $A$ which is lattice ordered in such a way that if $a \wedge b=0$ then $a c \wedge b=0$ for all $c \in A^{+}$.

An $f$-ring without nonzero nilpotents is an $f^{*}$-ring. For if $b, c \in A$ and $b \wedge c=0$ then $b c \wedge b c=0$, that is $b c=0$. So for any $a \notin A^{+}$, $s \in A^{+}$and $n \in Z^{++}, s a-a^{2 n}=s a^{+}-s a^{-}-\left(a^{+}\right)^{2 n}-\left(a^{-}\right)^{2 n} \leqq s a^{+}-\left(a^{-}\right)^{2 n}$. And the latter expression is not in $A^{+}$since $a^{+} \wedge a^{-}=0$ yields $s a^{+} \wedge\left(a^{-}\right)^{2 n}=0$; whence $\left(s a^{+}-\left(a^{-}\right)^{2 n}\right)^{-}=\left(a^{-}\right)^{2 n} \neq 0$. Furthermore if $A$ is an $f^{*}$-ring which is lattice ordered and such that $a \wedge b=0$ implies $a b=0$ then for any $P \in \mathscr{P},(a \wedge b)(P)=a(P) \wedge b(P)$. For if $a \wedge b=c$ then $(a-c) \wedge(b-c)=0$, so $(a-c)(b-c)=0$; whence $(a-c)(P)(b-c)(P)=0$. But $A / P$ is a ring with integrity, so $(a-c)(P)=0$ or $(b-c)(P)=0$. Therefore, since $(a-c) \geqq 0$ and $(b-c) \geqq 0$, $(a-c)(P) \wedge(b-c)(P)=0$ and $a(P) \wedge b(P)=c(P)=(a \wedge b)(P)$. Consequently the isomorphisms set up in Theorems 1 and 2 are isomorphisms onto a lattice ordered subdirect union of toi-rings which preserve lattice relations.

As for Theorem 3, it follows that for any $a, b \in A$ and any $M \in \mathscr{C}^{* * *}, \bar{a}(M) \wedge \bar{b}(M)=\bar{a} \wedge \bar{b}(M)$. Whence the sets $\left\{\mathscr{C}^{* * *}(a>0)\right\}_{a \in A}$ form a basis for $\mathscr{T}$ and so does the class of sets $\left\{\mathscr{C}^{* * *}(\bar{a}>0)\right\}_{a \in A}$. So each function $a$ is finite on a dense subset of $\mathscr{C}^{* * *}$ (i.e. it is an extended function in the sense of [2]). Finally, Lemma 2.6 (ii) of [2] may be used to prove that the topology of $\mathscr{C}^{* * *}$ is precisely the weak topology induced by the bounded functions in $\bar{A} \mid \mathscr{L}^{* * *}$.

Note added in proof. Lemma 3, together with the remark at the end of the fourth paragraph of $\S 3$, shows that for any toi ring, $R$, the following three properties are equivalent:

(i) $R$ is $r$-Archimedean,

(ii) $R$ has no nonzero $r$-infinitesimal elements,

(iii) Every element of $R^{++}$is an $r$-order unit.

So Theorem 2 can be sharpened. For example, we may omit "with no nonzero $r$-infinitesimal elements". 


\section{REFERENCES}

1. G. Birkhoff, and R. S. Pierce, Lattice ordered rings, An. Acad. Brasil Ci., 28 (1956),. 41-69.

2. D. J. Johnson, A representation theory for a class of Archimedean lattice ordered rings, Proc. London Math. Soc., 12 (1962), 207-225.

3. J. L. Kelley, General Topology, Van Nostrand. (New York 1955).

4. R. S. Pierce, Radicals in function rings, Duke Math. J., 23 (1956), 253-261.

PURDUE UNIVERSITY 


\section{PACIFIC JOURNAL OF MATHEMATICS}

\section{EDITORS}

\author{
Robert Osserman \\ Stanford University \\ Stanford, California
M. G. Arsove
University of Washington
Seattle 5 , Washington

\author{
J. DugundJI \\ University of Southern California \\ Los Angeles 7, California
}

Lowell J. Paige

University of California

Los Angeles 24, California

\section{ASSOCIATE EDITORS}
E. F. BECKENBACH
B. H. NeumanN
F. WOLF
K. YOSIDA

\section{SUPPORTING INSTITUTIONS}

\author{
UNIVERSITY OF BRITISH COLUMBIA \\ CALIFORNIA INSTITUTE OF TECHNOLOGY \\ UNIVERSITY OF CALIFORNIA \\ MONTANA STATE UNIVERSITY \\ UNIVERSITY OF NEVADA \\ NEW MEXICO STATE UNIVERSITY \\ OREGON STATE UNIVERSITY \\ UNIVERSITY OF OREGON \\ OSAKA UNIVERSITY \\ UNIVERSITY OF SOUTHERN CALIFORNIA
}

\author{
STANFORD UNIVERSITY \\ UNIVERSITY OF TOKYO \\ UNIVERSITY OF UTAH \\ WASHINGTON STATE UNIVERSITY \\ UNIVERSITY OF WASHINGTON \\ * * * * \\ AMERICAN MATHEMATICAL SOCIETY \\ CALIFORNIA RESEARCH CORPORATION \\ SPACE TECHNOLOGY LABORATORIES \\ NAVAL ORDNANCE TEST STATION
}

Mathematical papers intended for publication in the Pacific Journal of Mathematics should by typewritten (double spaced), and on submission, must be accompanied by a separate author's résumé. Manuscripts may be sent to any one of the four editors. All other communications to the editors should be addressed to the managing editor, L. J. Paige at the University of California, Los Angeles 24, California.

50 reprints per author of each article are furnished free of charge; additional copies may be obtained at cost in multiples of 50 .

The Pacific Journal of Mathematics is published quarterly, in March, June, September, and December. Effective with Volume 13 the price per volume (4 numbers) is $\$ 18.00$; single issues, $\$ 5.00$. Special price for current issues to individual faculty members of supporting institutions and to individual members of the American Mathematical Society: $\$ 8.00$ per volume; single issues $\$ 2.50$. Back numbers are available.

Subscriptions, orders for back numbers, and changes of address should be sent to Pacific Journal of Mathematics, 103 Highland Boulevard, Berkeley 8, California.

Printed at Kokusai Bunken Insatsusha (International Academic Printing Co., Ltd.), No. 6, 2-chome, Fujimi-cho, Chiyoda-ku, Tokyo, Japan.

PUBLISHED BY PACIFIC JOURNAL OF MATHEMATICS, A NON-PROFIT CORPORATION

The Supporting Institutions listed above contribute to the cost of publication of this Journal, but they are not owners or publishers and have no responsibility for its content or policies. 


\section{Pacific Journal of Mathematics}

\section{Vol. 14, No. $3 \quad$ July, 1964}

Erik Balslev and Theodore William Gamelin, The essential spectrum of a class of ordinary differential operators . . . . . . . . . . . . . . . . . . . .

James Henry Bramble and Lawrence Edward Payne, Bounds for derivatives in

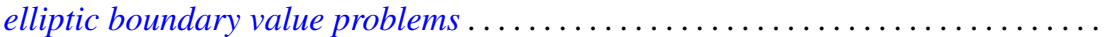

Hugh D. Brunk, Integral inequalities for functions with nondecreasing

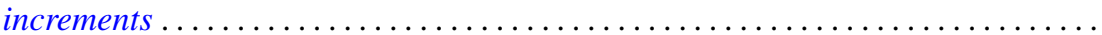

William Edward Christilles, A result concerning integral binary quadratic

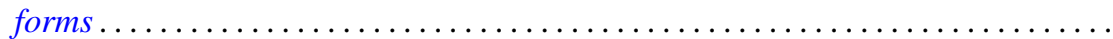

Peter Crawley and Bjarni Jónsson, Refinements for infinite direct decompositions of

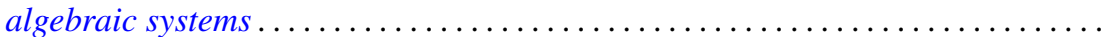

Don Deckard and Carl Mark Pearcy, On continuous matrix-valued functions on a Stonian space.

Raymond Frank Dickman, Leonard Rubin and P. M. Swingle, Another

characterization of the $n$-sphere and related results $\ldots \ldots \ldots \ldots \ldots \ldots$

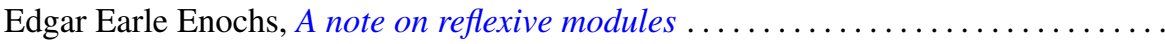

Vladimir Filippenko, On the reflection of harmonic functions and of solutions of the

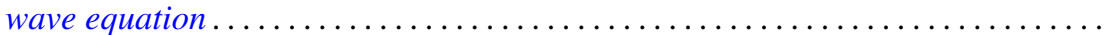

Derek Joseph Haggard Fuller, Mappings of bounded characteristic into arbitrary

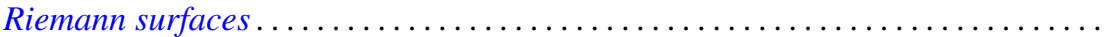
895

Curtis M. Fulton, Clifford vectors . . . . . . . . . . . . . . . . . . . . . . . . . . . . . . 917

Irving Leonard Glicksberg, Maximal algebras and a theorem of Radó . .

919

Kyong Taik Hahn, Minimum problems of Plateau type in the Bergman metric

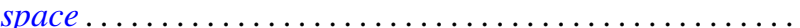

A. Hayes, A representation theory for a class of partially ordered rings...

J. M. C. Joshi, On a generalized Stieltjes trasform

J. M. C. Joshi, Inversion and representation theorems for a generalized Laplace transform ...

Eugene Kay McLachlan, Extremal elements of the convex cone $B_{n}$ of functions ...

Robert Alan Melter, Contributions to Boolean geometry of p-rings ...

James Ronald Retherford, Basic sequences and the Paley-Wiener criterion . . . . . . . 1019

Dallas W. Sasser, Quasi-positive operators. .

Oved Shisha, On the structure of infrapolynomials with prescribed coefficients ..

Oved Shisha and Gerald Thomas Cargo, On comparable means

Maurice Sion, A characterization of weak ${ }^{*}$ convergence ........

Morton Lincoln Slater and Robert James Thompson, A permanent inequality for

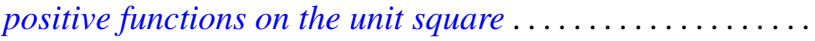

David A. Smith, On fixed points of automorphisms of classical Lie algebras ...

Sherman K. Stein, Homogeneous quasigroups ................

J. L. Walsh and Oved Shisha, On the location of the zeros of some infrapolynomials with prescribed coefficients .

Ronson Joseph Warne, Homomorphisms of $d$-simple inverse semigroups with identity . 